\title{
Reason Analysis for the increase of single-phase short circuit current and its suppressing measure
}

\author{
Y. $\mathrm{Li}^{1}, \mathrm{H} . \mathrm{ZHANG}^{1}, \mathrm{G} . \mathrm{Chen}^{1}$ \\ ${ }^{1}$ State Grid Sichuan Electric Power Research Institute, Chengdu, China
}

KEYWORD: Single-phase short circuit current; Neutral grounding; Small reactance; Autotransformer

ABSTRACT: Aiming at the issue that the single-phase short circuit current at the $220 \mathrm{kV}$ bus of Chengdu 500kV substation exceeds the three-phase short current or even exceeds the breaking capacity of breaker, the reason is analyzed and the measure that main transformer neutral grounding by small reactance is put forward to reduce the single-phase short circuit. According to the planning power network of Sichuan, this paper analyzes the limiting effects of $500 \mathrm{kV}$ autotransformer neutral grounding by small reactance on $220 \mathrm{kV}$ single-phase short circuit current by simulation and calculation. This paper also analyzes the influence of neutral grounding by small reactance on insulation level of neutral point, and put forward a directive idea about small reactor's selection .

\section{Introduction}

Along with the extension of power system and the growth of power load, the short circuit current level is increasingly improved and being an important factor restricting the network development ${ }^{[1-2]}$. Considering the economic factor, autotransformer is widely used in $500 \mathrm{kV}$ and above power system at present. Because of autotransformer neutral directly grounding, the zero sequence reactance decreases which result in the rapid increase of the single-phase short circuit current at the $220 \mathrm{kV}$ bus of $500 \mathrm{kV}$ substation. In severe it will exceeds the three-phase short current or even exceeds the breaker's breaking capacity of 50kA at medium voltage side ${ }^{[3-6]}$.

The basic measure to control the short circuit current is reasonable power network planning. For the existing system, two measures to restrict the single-phase short circuit current are often adopted. One measure is that $220 \mathrm{kV}$ split bus arrangement which will decease power supply reliability; the other measure is main transformer neutral point grounding through small reactance which has advantages in small investment and high validity of decreasing the single-phase short circuit current ${ }^{[7-9]}$.

In this paper the reason of rapid increase of single-phase short circuit current in $500 \mathrm{kV}$ substation is analyzed according to the transformer's characteristics of Chengdu. Longwang substation and Xindu substation are selected and the Power System Analysis Software Package (PSASP) is used to make short-circuit current calculation of main transformer neutral grounding via low resistance. This paper analyzes the limiting effects of $500 \mathrm{kV}$ autotransformer neutral grounding by small reactance on $220 \mathrm{kV}$ single-phase short-circuit current and the influence of insulation level of neutral point. This paper also puts forward a directive idea about small reactor's selection.

\section{Reason analysis of single-phase short circuit current exceeding three-phase short current}

Generally think the positive sequence reactance is equal to the negative sequence reactance in power system. Single-phase short circuit current can be represented as follows:

$$
I_{k 1}=\frac{3 U_{k}}{X_{\Sigma 1}+X_{\Sigma 2}+X_{\Sigma 0}}=\frac{3 U_{k}}{2 X_{\Sigma 1}+X_{\Sigma 0}}
$$

Three-phase short-circuit current can be represented as follows:

$$
I_{k 3}=\frac{U_{k}}{X_{\Sigma 1}}
$$

Among them, $U_{k}$ represents the voltage prior to fault; $X_{\Sigma l}, X_{\Sigma l}$ and $X_{\Sigma^{0}}$ respectively represent the positive sequence reactance, the negative sequence reactance and the zero sequence reactance. 
In term of the two equations above we can know that when $X_{\Sigma 0^{0}}<X_{\Sigma l}$, the single-phase short circuit current exceeds the three-phase short current.

At present, all the $500 \mathrm{kV}$ transformers and a small part of $220 \mathrm{kV}$ transformers of Chengdu power network are autotransformers, and all the step-down transformers are three-winding transformers with the third winding triangle connecting. The simulation according to the planning power network of Sichuan shows that part of single-phase short circuit current at the $220 \mathrm{kV}$ bus exceeds the threephase short current or even exceeds the breaking capacity of breaker, in which the single-phase short circuit current at $220 \mathrm{kV}$ bus of $500 \mathrm{kV}$ substation is the most serious. the general view about this is that the zero sequence reactance decreases because of autotransformer neutral directly grounding. Through further study it is found that the positive sequence circuit of transformer with the third winding triangle connecting is different from the zero sequence circuit. The positive sequence circuit of the third winding is open circuit and the zero sequence circuit is short circuit. So the zero sequence network has more grounding branches which causes the zero sequence equivalent impedance maybe smaller than positive sequence equivalent impedance.

Figure 1 shows the positive sequence and the zero sequence equivalent network at the mediumvoltage side of three-winding transformer. $X_{H 1}, X_{M 1}, X_{L 1}, X_{H 0}, X_{M 0}$ and $X_{L 0}$ respectively represent the positive sequence and the zero sequence reactance at the high-voltage, medium-voltage and lowvoltage side of transformer. $X_{S I}$ and $X_{S 0}$ respectively represent the positive sequence and zero sequence equivalent reactance at $500 \mathrm{kV}$ system side. $X_{F 1}$ and $X_{F 0}$ respectively represent the positive sequence and zero sequence equivalent reactance at $220 \mathrm{kV}$ system side. The positive sequence equivalent reactance $X_{K 1}$ and the zero sequence equivalent reactance $X_{K 0}$ of the system from $220 \mathrm{kV}$ side of transformer can be represented as follows according to figure 1:

$$
\begin{aligned}
& x_{K 1}=X_{F 1} / /\left(X_{H 1}+X_{M 1}+X_{S 1}\right) \\
& X_{K 0}=X_{F 0} / /\left(\left(X_{H 0}+X_{S 0}\right) / / X_{L 0}+X_{M 0}\right)
\end{aligned}
$$

For the $Y N, y n, d$ transformer in load center, the positive sequence reactance is equal to the zero sequence reactance. Moreover, the positive sequence network structure and the zero sequence network structure at both $500 \mathrm{kV}$ system side and $220 \mathrm{kV}$ system side are almost the same because of star connection mode and neutral directly grounding of autotransformer at both high-voltage side and medium-voltage side ${ }^{[10]}$. generally, the zero line parameter is 3-5 times of positive line parameter, so $X_{S 0}>X_{S 1}, X_{F 0}>X_{F 1}$. From the comparison of two equations above it can be known that zero sequence equivalent reactance of the system from $220 \mathrm{kV}$ side of transformer maybe smaller than positive sequence equivalent reactance because of the existence of $X_{L 0}$, which result in the single-phase short circuit current at the $220 \mathrm{kV}$ bus exceeding the three-phase short current.

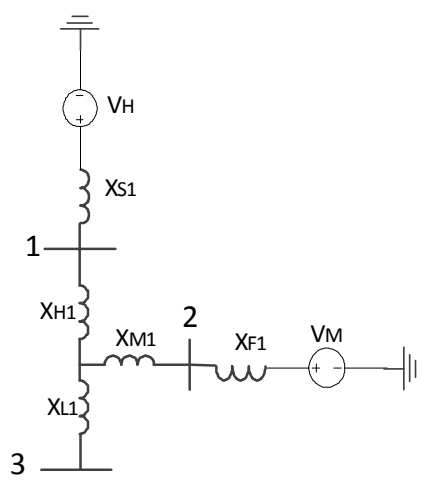

(a) Positive sequence equivalent network

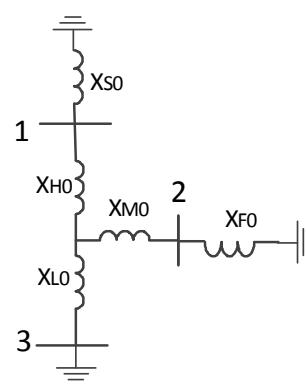

(b) zero sequence equivalent network

Figure 1. Positive sequence equivalent network and zero sequence equivalent network at $500 \mathrm{kV}$ substations 
Taking the Longwang substation in Chengdu power grid of 2016 as example, calculation and verification are carried out on the reason that the single-phase short circuit current at the $220 \mathrm{kV}$ bus of $500 \mathrm{kV}$ substation exceeding the three-phase short current. After electromagnetic loop untying of Chengdu power network, the calculated results show that the three-phase short current at the 220 $\mathrm{kV}$ bus of Longwang $500 \mathrm{kV}$ substation is $48.44 \mathrm{kA}$, and the single-phase short circuit current is $56.13 \mathrm{kA}$, which is $7.69 \mathrm{kA}$ larger.

According to the results, the single-phase short circuit current at the $220 \mathrm{kV}$ bus of Longwang substation is $2.53 \mathrm{kA}$ larger than the three-phase short current with \#1 main transformer of Longwang unloaded (condition 1). However, the single-phase short circuit current is $0.19 \mathrm{kA}$ smaller than the three-phase short current when the third winding is furthermore disconnected (condition 2). The results show that the zero sequence equivalent reactance of the system from $220 \mathrm{kV}$ side of transformer is smaller than the positive sequence equivalent reactance without considering the third winding of main transformer, and the conclusion is just opposite while considering the third winding. Furthermore, if Longwang substation supply power for Tuanjie substation by single circuit line with both \#1 main transformer of Longwang and main transformers of Tuanjie unloaded, the single-phase short circuit current at the $220 \mathrm{kV}$ bus of Longwang substation is $2.84 \mathrm{kA}$ larger than the three-phase short current (condition 3). And the single-phase short circuit current is $0.2 \mathrm{kA}$ smaller than the three-phase short current when the third winding of both \#1 main transformer of Longwang and main transformers of Tuanjie are disconnected and the transformers are unloaded (condition 4).

It can be concluded that the third winding of $220 \mathrm{kV}$ substation also decreases the zero sequence equivalent reactance of the system.

The short current at the $220 \mathrm{kV}$ bus of Longwang substation is calculated in different conditions and the results are shown as Table 1.

Table 1. Influence analysis of the third winding for the single-phase short circuit current

\begin{tabular}{llll}
\hline Condition & $\begin{array}{l}\text { Three-phase } \\
\text { short circuit } \\
\text { current }\end{array}$ & & $\begin{array}{l}\text { Single-phase } \\
\text { short circuit } \\
\text { current }\end{array}$ \\
\cline { 2 - 2 } $\mathrm{kA}$ & 16.51 & 19.04 \\
\hline 1 & 16.51 & 16.32 \\
3 & 16.50 & 19.34 \\
4 & 16.50 & 16.30 \\
\hline
\end{tabular}

Therefore, because of the two reasons: one is the widely use of autotransformer, the other is the difference between the positive sequence circuit and the zero sequence circuit of transformers with the third winding triangle, the single-phase short circuit current at the $220 \mathrm{kV}$ bus of Chengdu $500 \mathrm{kV}$ substation exceeds the three-phase short current. For the second reason, not only the triangle winding of $500 \mathrm{kV}$ transformers but also that of $220 \mathrm{kV}$ transformers has the similar effect of increasing the single-phase short circuit current.

\section{Limiting effects of autotransformer neutral grounding by small reactance on single-phase short-circuit current}

The Limiting effects on single-phase short-circuit current of autotransformer neutral grounding by small reactance with different values are calculated underside.

Taking Longwang and Xindu piece area of Chengdu power grid after electromagnetic loop untying as example. The analysis results are shown in Table 2 and Figure 2. 
Table 2. Single-phase short circuit current at the $220 \mathrm{kV}$ bus of Longwang and Xindu with autotransformers neutral grounding by small reactance of different values

\begin{tabular}{|c|c|c|c|}
\hline $\begin{array}{l}\text { Serial } \\
\text { num- } \\
\text { ber }\end{array}$ & $\begin{array}{l}\text { Neutral } \\
\text { reactor } \\
\text { value }\end{array}$ & $\begin{array}{l}\text { Long- } \\
\text { wang } \\
\text { substa- }\end{array}$ & $\begin{array}{l}\text { Xindu } \\
\text { substa- } \\
\text { tion }\end{array}$ \\
\hline & $\Omega$ & $\mathrm{kA}$ & $\mathrm{kA}$ \\
\hline 1 & 0 & 53.93 & 48.80 \\
\hline 2 & 2 & 51.32 & 45.55 \\
\hline 3 & 4 & 49.61 & 43.39 \\
\hline 4 & 6 & 48.39 & 41.85 \\
\hline 5 & 8 & 47.48 & 40.70 \\
\hline 6 & 10 & 46.77 & 39.79 \\
\hline 7 & 12 & 46.2 & 39.07 \\
\hline 8 & 14 & 45.73 & 38.47 \\
\hline 9 & 16 & 45.33 & 37.98 \\
\hline 10 & 18 & 45.00 & 37.55 \\
\hline 11 & 20 & 44.71 & 37.19 \\
\hline
\end{tabular}

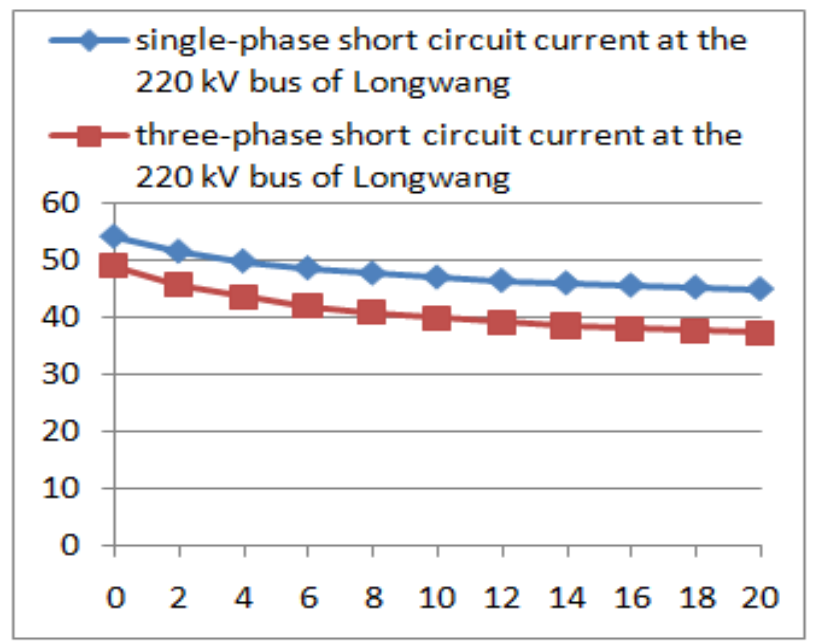

Figure 2. Limiting effects on single-phase short-circuit current of autotransformer neutral grounding by small reactance of different values.

From the results it is known that with the gradually increasing of the small reactor value at each autotransformer's neutral of Longwang and Xindu, the limiting effect on single-phase short-circuit current reaches a saturation value, and the overvoltage of transformer's neutral point increases. Therefore, based on comprehensive consideration of the effect of reducing single-phase short circuit current and the withstand voltage level of transformer's neutral point, the suitable value of neutral reactor is $10 \sim 15 \mathrm{ohm}$. This paper selects $14 \mathrm{ohm}$.

Next this paper analyzes the influence of neutral grounding by small reactance on insulation level of neutral point. At present, the power frequency over-voltage withstanding level of main transformer's neutral point of Chengdu 500kV substation is $140 \mathrm{kV}$. Taking Longwang and Xindu piece area of Chengdu power grid as example, the overvoltage is calculated after each transformer neutral grounding by small reactance of $14 \mathrm{ohm}$ at Longwang substation and Xindu substation. The results are shown in Table 3.

Table 3. Neutral point voltage and current after $220 \mathrm{kV}$ bus fault of Longwang substation

\begin{tabular}{lllll}
\hline Fault type & condition & Neutral point voltage & & Neutral point current \\
& & $\mathrm{kV}$ & $\mathrm{kA}$ \\
\hline Single phase & Before adding reactor & 0.0 & 10.0 \\
fault & After adding reactor & 43.8 & 3.2 \\
Phase-to -phase & Before adding reactor & 0.0 & 10.9 \\
fault & After adding reactor & 42.4 & 2.9 \\
\hline
\end{tabular}


It can be known that after adding the reactor of $14 \mathrm{ohm}$, the maximum neutral point voltage of main transformers at Longwang substation is $43.8 \mathrm{kV}$ under asymmetric fault. This value is lower than the over-voltage withstanding level and has high security margin.

\section{Conclusion}

(1)This paper analyzes the two reasons for the increasing of the single-phase short circuit current at $220 \mathrm{kV}$ bus of $500 \mathrm{kV}$ substation. One is the widely use of autotransformer with the neutral directly grounding; the other is the difference between the positive sequence circuit and the zero sequence circuit of transformers with the third winding triangle connecting.

(2)According to the high load data of Sichuan Power Grid in 2016, this paper selects Longwang substation and Xindu substation in Chengdu Power Grid to make short-circuit current calculation with main transformer neutral grounding by small reactance. The results show that $500 \mathrm{kV}$ autotransformer neutral grounding by small reactance is effective to decrease the $220 \mathrm{kV}$ single-phase shortcircuit current. Therefore autotransformer neutral grounding by small reactance is suggested as the main measure to solve the problem of single-phase short circuit current exceeding the breaker's breaking capacity. However, with the gradually increasing of the small reactor value, the limiting effect on single-phase short circuit current reaches a saturation value. Therefore, the suitable value of neutral reactor should be selected based on comprehensive consideration of the effect of reducing single-phase

(3)According to calculation, the voltage deviation of neutral point caused by autotransformer neutral grounding by small reactance is within the insulation level of neutral point and has high security margin.

\section{References}

[1] Wang, F. \& Li, Q. 2007. Short-circuit current control for Beijing-Tianjin-Tangshan grid. North China Electric Power (4): 17-19

[2] Yuan, J. \& Liu W. Y., et a1. 2007. Application of measures limiting short circuit current in northwest China power grid. Power System Technology 31(10): 42-45.

[3] Zhang, H. \& Gan, D.Q. 2009. Analysis of application of $500 \mathrm{kV}$ transformer with low reactance grounding. Electric Power Construction 29(11): 38-40.

[4] Zhu, T. Y. 1999. Application of autotransformer neutral grounding by small reactance in 500 kV power system. Power System Technology 23(4): 15-18.

[5] Yang, X.P. \& Li, L., et a1. 2009. Running programs of limiting $500 \mathrm{kV}$ short-circuit current in Guangdong power grid. Automation of Electric Power System 33(7): 104-107.

[6] Hu, Z.G. \& W, F. 2007. Analysis of Transformer DC Bias Blocking Device and Its Impact on Relay Protection of AC System. Guangdong Electric Power 20(10): 20-23 .

[7] Zhu, J. \& Hu, H. et a1. 2006. Short circuit current of $500 \mathrm{kV}$ east China power grid and its limitation. East China Electric Power 34(7): 55-59.

[8] WU, D. 2005.Research on limiting large grid short-circuit current level measures. Hangzhou: Zhejiang University, 2005.

[9] LI, H. \& HUANG, H. \& ZHANG, Q. 2009. Study of limiting single phase ground short-current in Jiangsu power grid. Jiangsu Electrical Engineering 28(1): 19-21.

[10] Zheng, Y.C. \& Pan, J. 2001.A study of Relation Between zero and positive sequence impedances of large transformer. Hebei Electric Power 1(20): 4-6. 